\title{
Particle acceleration in shearing flows: the case for large-scale jets
}

\author{
Jie-Shuang Wang, ${ }^{1 \star}$ Brian Reville, ${ }^{2}$ Ruo-Yu Liu, ${ }^{3,4}$ Frank M. Rieger, ${ }^{2,5}$ Felix A. Aharonian ${ }^{2,6,7}$ \\ ${ }^{1}$ Tsung-Dao Lee Institute, Shanghai Jiao Tong University, Shanghai 200240, China \\ ${ }^{2}$ Max-Planck-Institut für Kernphysik, Saupfercheckweg 1, D-69117 Heidelberg, Germany \\ ${ }^{3}$ School of Astronomy and Space Science, Nanjing University, Nanjing 210093, China \\ ${ }^{4}$ Key laboratory of Modern Astronomy and Astrophysics (Nanjing University), Ministry of Education, Nanjing 210023, China \\ ${ }^{5}$ Institute of Theoretical Astrophysics, University of Heidelberg, Philosophenweg 12, D-69120 Heidelberg, Germany \\ ${ }^{6}$ Dublin Institute for Advanced Studies, 31 Fitzwilliam Place, Dublin 2, Ireland \\ ${ }^{7}$ High Energy Astrophysics Laboratory, RAU, 123 Hovsep Emin St Yerevan 0051, Armenia
}

\begin{abstract}
X-ray observations of kilo-parsec scale jets indicate that a synchrotron origin of the sustained non-thermal emission is likely. This requires distributed acceleration of electrons up to near PeV energies along the jet. The underlying acceleration mechanism is still unclear. Shear acceleration is a promising candidate, as velocity-shear stratification is a natural consequence of the collimated flow of a jet. We study the details of shear acceleration by solving the steady-state Fokker-Planck-type equation and provide a simple general solution for trans-relativistic jets for a range of magnetohydrodynamic turbulent power-law spectra. In general, the accelerated particle population is a power-law spectrum with an exponential-like cut-off, where the power-law index is determined by the turbulence spectrum and the balance of escape and acceleration of particles. Adopting a simple linearly decreasing velocity profile in the boundary of large-scale jets, we find that the multi-wavelength spectral energy distribution of X-ray jets, such as Centaurus A and 3C 273, can be reproduced with electrons that are accelerated up to $\sim \mathrm{PeV}$. In kpc-scale jets, protons may be accelerated up to $\sim \mathrm{EeV}$, supporting the hypothesis that large-scale jets are strong candidates for ultra-high-energy-cosmic-ray sources within the framework of shear acceleration.
\end{abstract}

Key words: acceleration of particles - galaxies: jets - X-rays: galaxies - gamma-rays: galaxies - radiation mechanisms: non-thermal

\section{INTRODUCTION}

Jets of active galactic nuclei (AGNs) can transport mass and energy from accreting super-massive black holes at the galaxy's center, feeding large-scale lobes that shine across the electromagnetic spectrum. In Fanaroff-Riley (FR) type galaxies, giant radio lobes, inflated by jets, are observed even on Mpc-scale (Fanaroff \& Riley 1974), implying that they can influence the intracluster medium significantly. In FR I jets, the region with higher radio-brightness is closer to the nucleus, while in FR II sources, the jet typically terminates at a working surface far from the core. The large-scale FR jets can also be bright in optical and X-ray bands, which makes them perfect candidates to explore jet dynamics, energy dissipation, and radiation processes (see Blandford et al. 2019, for a recent review). To date, approximately one hundred jets have been observed in X-rays (see Harris \& Krawczynski 2006; Schwartz et al. 2014, for reviews). Although the dominant structures are bright knots and terminal hotspots, quasi-continuous emission is observed in some X-ray jets, such as Centaurus A (Kraft et al. 2002; Kataoka et al. 2006).

In FR I jets, the radio, optical, and X-ray spectrum can typically be explained by synchrotron radiation from a single population of electrons (e.g., Perlman et al. 2001; Hardcastle et al. 2001; Sun et al. 2018). The X-ray measurements of FR II jets can however exhibit

^ jiesh.wang@gmail.com

(c) The Authors much harder spectra (e.g., Jester et al. 2006, 2007). This has motivated studies suggesting that in FR II jets X-rays are produced via inverse Compton (IC) scattering by low-energy electrons (around tens of $\mathrm{MeV}$ ) off cosmic microwave background (CMB) photons, while relativistic jets are required with bulk Lorentz factors of order 10 (Tavecchio et al. 2000; Celotti et al. 2001). Although both emission mechanisms can account for the X-ray spectral energy distribution (SED), they have different predictions. Comparing with a synchrotron radiation model, the IC/CMB model will lead to a higher gamma-ray flux, but less polarization (e.g. Uchiyama 2008). Corresponding tests have been performed and favour the synchrotron origin (see Georganopoulos et al. 2016; Perlman et al. 2020, for recent reviews), which includes the optical polarimetry observation of PKS 1136-135 (Cara et al. 2013), 3C 273, PKS 0637-752, and PKS 1150+497 (Perlman et al. 2020), and gamma-ray studies of 3C 273 (Meyer \& Georganopoulos 2014), PKS 0637-752 (Meyer et al. 2015), PKS 1136-135, PKS 1229-021, PKS 1354+195, and PKS 2209+080 (Breiding et al. 2017). With the IC origin of X-ray emission in these sources now disfavoured, it is reasonable to explore a synchrotron origin in other jets and its physical implications. The electrons radiating in X-rays suffer substantial synchrotron cooling losses on a timescale of decades, such that for a localised acceleration site, the X-ray-bright region should not extend more than tens of parsecs, contrary to observations. As such, a distributed in-situ (re-)acceleration mechanism is required for large-scale X-ray jets.

Several candidate acceleration mechanisms have been pro- 
posed for particle acceleration in jets (see Blandford et al. 2019; Matthews et al. 2020, for recent reviews). Shock acceleration, being arguably the best understood, is commonly invoked as the primary acceleration mechanism in jets, motivated by its successful application in many other astrophysical scenarios, such as supernova remnants (e.g., Drury 1983; Blandford \& Eichler 1987; Bell 2013). While acceleration of electrons to high energies at shocks is possible, it only occurs where strong shock formation is possible, for example re-collimation shocks or the jet termination shock. For magnetically dominated jets, relativistic magnetic reconnection, possibly driven by the kink instability (e.g. Begelman 1998), has been suggested as a driver for particle acceleration. This process has been demonstrated to be efficient in particle-in-cell (PIC) simulations (e.g., Sironi \& Spitkovsky 2014; Werner et al. 2016). However, beyond the kpc-scale, jets are generally kinetically dominated (e.g., Sikora et al. 2005; Potter 2017; Chatterjee et al. 2019), and as such, an alternative mechanism that can accelerate particles continuously over tens of kiloparsecs is needed. Stochastic acceleration in a turbulent jet boundary layer is a possible alternative (e.g., Stawarz \& Ostrowski 2002), though rapid acceleration requires a high Alfvén speed in the jet (O'Sullivan et al. 2009). For typical conditions of $1-100 \mathrm{kpc}$ scale jets, Liu et al. (2017) argue that stochastic acceleration may not be efficient enough to overcome the cooling and escape of particles, and hence may have difficulties to produce X-ray emitting electrons.

Shear acceleration, another type of Fermi acceleration mechanism, can in principle accelerate high-energy electrons in the steep velocity gradients that inevitably develop at the boundary of jets (see e.g., Berezhko 1981; Berezhko \& Krymskii 1981; Earl et al. 1988 for early studies and Rieger 2019 for a recent review). Indeed, high-resolution radio imaging and polarization studies of large-scale jets indicate the presence of such velocity gradients transverse to the main jet axes (e.g., Laing \& Bridle 2014; Gabuzda et al. 2014; Nagai et al. 2014; Boccardi et al. 2016). Numerical simulations have further shown that the global stability of jets also depends on the properties of the shear layer (e.g., Mizuno 2013; Kim et al. 2018). In gradual shearing flows, particles gain/lose energy by elastically scattering off magnetic field inhomogeneities (e.g., Rieger \& Duffy 2016; Webb et al. 2018), which are considered to be frozen-in to the corresponding velocity-shear layers, i.e. the particle energy is approximately conserved in the local fluid frame. For shear acceleration in kpc-scale jets, under favourable conditions, the accelerated particles are expected to form power-law spectra, with cut-offs predicted at $\sim$ $\mathrm{EeV}$ for protons, although cooling limits electrons to $\sim \mathrm{PeV}$ energies (e.g., Rieger \& Duffy 2019, 2021). To this end, Liu et al. (2017) have shown that the accelerated electrons can in principle emit X-ray up to $100 \mathrm{keV}$ with a hard spectrum via synchrotron radiation, while the diffusive escape of accelerated electrons can soften the spectrum. The joint effect of shear acceleration and diffusive escape results in a large range for the accelerated electron spectral slope, but the influence on the predicted X-ray spectrum in large-scale jets has yet to be quantified.

In this paper, we explore particle acceleration in the gradual shear flows at the boundary of large-scale jets by solving a Fokker-Plancktype equation with an analytical approach, where the acceleration, cooling, and diffusive escape terms are included simultaneously. The steady-state solution is derived in Section 2. In Section 3, we study the corresponding radiation from the accelerated particles and apply it to the observed large-scale jets. Exemplary modellings of the SEDs in Centaurus A and 3C 273 are presented. In Section 4, we explore the capability of acceleration of cosmic rays (CRs) in large-scale jets. The conclusion and discussion are presented in Section 5.

\section{ACCELERATED PARTICLE SPECTRA IN SHEARING FLOWS}

In fast shearing flows, particles can gain energy by elastically scattering off magnetic field inhomogeneities embedded in the local fluid. This can be understood as a stochastic Fermi-type acceleration mechanism (e.g. Rieger et al. 2007). We consider first electrons. Taking these to be magnetised, the mean scattering time can be formulated as (see for example Schlickeiser 2002),

$\tau_{\mathrm{sc}}=\xi_{1}^{-1}\left(\frac{r_{\mathrm{L}}}{\Lambda_{\max }}\right)^{1-q} \frac{r_{\mathrm{L}}}{c} \equiv A_{0} \gamma^{2-q}$,

where $\xi_{1}=\delta B^{2} / B_{0}^{2}$ denotes the energy density ratio of turbulent field $(\delta B)$ to the mean magnetic field $\left(B_{0}\right), r_{\mathrm{L}}=\gamma m_{\mathrm{e}} c^{2} / e B_{0}$ is the Larmor radius of an electron with Lorentz factor $\gamma$ and $A_{0}=$ $\xi_{1}^{-1}\left(\Lambda_{\max } / c\right)^{q-1}\left(m_{e} c / e B_{0}\right)^{2-q}$. The index $q$ denotes the power-law index of the turbulent spectrum, e.g., $q=5 / 3$ for a Kolmogorov-type turbulence, $q=3 / 2$ for Kraichnan-type, and $q=1$ for Bohm-type. $\Lambda_{\max }$ is the outer turbulence scale. If the turbulent field dominates over the mean field, provided $q>1$, it is reasonable to replace the mean field with the strength at the largest scales, i.e. take $B_{0} \approx \delta B$ and $\xi_{1} \approx 1$. We here focus on the range of $1 \leq q \leq 2$, and note that Eq. (1) only applies if $r_{\mathrm{L}}<\Lambda_{\max }$. Beyond this scale, the scattering time will follow a different behaviour.

The energy space diffusion coefficient in a gradual shear flow is given by (e.g., Rieger \& Duffy 2006)

$\left\langle\frac{\Delta \gamma^{2}}{\Delta t}\right\rangle_{\mathrm{sh}} \simeq D_{\mathrm{sh}} \gamma^{2} \tau_{\mathrm{sc}} \equiv A_{1} \gamma^{4-q}$,

where $\langle\ldots\rangle$ denotes averaging over an isotropic particle distribution. For a cylindrical outflow, the viscous momentum transfer coefficient is (e.g., Rieger \& Duffy 2004; Webb et al. 2018),

$D_{\mathrm{sh}}=\frac{1}{15} \Gamma_{\mathrm{j}}^{4}(r) c^{2}\left(\frac{\partial \beta_{\mathrm{j}}(r)}{\partial r}\right)^{2}$,

where $\Gamma_{\mathrm{j}}(r)=\left(1-\beta_{\mathrm{j}}^{2}\right)^{-1 / 2}$ and $\beta_{\mathrm{j}}(r) c$ are the bulk Lorentz factor and velocity of different layers located at a distance $r$ from the jet axis, and $A_{1}=A_{0} D_{\text {sh }}$. For a trans-relativistic flow and a linearly decreasing velocity profile, this expression essentially becomes independent of $r$.

The corresponding acceleration time is,

$\tau_{\mathrm{sh}}=\frac{2}{6-q} D_{\mathrm{sh}}^{-1} \tau_{\mathrm{sc}}^{-1}=\frac{2}{6-q} A_{1}^{-1} \gamma^{q-2}$.

In magnetised jets, the high-energy electrons will undergo continuous energy losses via synchrotron and IC radiation. Neglecting Klein-Nishina corrections, the cooling rate is

$\left\langle\dot{\gamma}_{\mathrm{c}}\right\rangle=\frac{\sigma_{T} \gamma^{2} B^{2}}{6 \pi m_{e} c}(1+f) \equiv A_{2} \gamma^{2}$,

where $\sigma_{T}$ is the Thomson scattering cross-section, and $f=U_{\text {rad }} / U_{B}$ is the energy density ratio between the target photon field $\left(U_{\mathrm{rad}}\right)$ for the IC process and the magnetic field $\left(U_{B}=B^{2} / 8 \pi\right)$. For IC/CMB scattering, $U_{\mathrm{CMB}}=4.18 \times 10^{-13}(1+z)^{4} \mathrm{erg} \mathrm{cm}^{-3}$ and hence for $B=$ $10^{-5} B_{-5} \mathrm{G}$, we find $f=0.1(1+z)^{4} B_{-5}^{-2}$. Synchrotron radiation will dominate over IC/CMB if $B>3.2(1+z)^{2} \mu \mathrm{G}$. Combining radiative losses and shear acceleration, the maximum energy is obtained from $\left\langle\dot{\gamma}_{\mathrm{c}}\right\rangle=\gamma / \tau_{\mathrm{sh}}$ for electrons, which yields

$\gamma_{\max }=\left(\frac{6-q}{2} \frac{A_{1}}{A_{2}}\right)^{1 /(q-1)}$. 
An energetic particle can also escape from the acceleration zone by diffusion, and the corresponding escape time is given by

$\tau_{\mathrm{esc}}=\frac{\Delta r_{\mathrm{j}}^{2}}{2 \kappa}=\frac{3 \Delta r_{\mathrm{j}}^{2}}{2 c^{2}} \tau_{\mathrm{sc}}^{-1} \equiv A_{3} \gamma^{q-2}$,

where $\Delta r_{\mathrm{j}}$ is the width of the shearing region of the jet, $\kappa=c^{2} \tau_{\mathrm{sc}} / 3$ is the particles' spatial diffusion coefficient, and $A_{3}=1.5 \Delta r_{\mathrm{j}}^{2} A_{0}^{-1} c^{-2}$. Note, that we assume isotropic diffusion. A strong guide field will inhibit escape, but also reduces the acceleration rate (Webb 1989).

Taking all these effects into consideration, the evolution of the particle distribution function $n(\gamma, t)$ can be expressed as a Fokker-Planck-type equation (e.g. Liu et al. 2017),

$$
\begin{aligned}
\frac{\partial n(\gamma, t)}{\partial t} & =\frac{1}{2} \frac{\partial}{\partial \gamma}\left[\left\langle\frac{\Delta \gamma^{2}}{\Delta t}\right\rangle \frac{\partial n(\gamma, t)}{\partial \gamma}\right] \\
& -\frac{\partial}{\partial \gamma}\left[\left(\frac{1}{\gamma}\left\langle\frac{\Delta \gamma^{2}}{\Delta t}\right\rangle-\left\langle\dot{\gamma}_{\mathrm{c}}\right\rangle\right) n(\gamma, t)\right]-\frac{n(\gamma, t)}{\tau_{\mathrm{esc}}}+Q(\gamma, t),
\end{aligned}
$$

where $Q(\gamma, t)$ denotes the injection rate.

From Eq. (4), it follows that for $q<2$ the acceleration time is a decreasing function of particle energy. This means that shear acceleration is in theory more efficient to accelerate higher-energy particles, although it likely requires seed particles to be accelerated to sufficiently high energies (Stawarz \& Ostrowski 2002; Rieger \& Duffy 2006). Several processes have been suggested for the acceleration of seed particles, including stochastic (classical 2nd order Fermi) acceleration (Liu et al. 2017), magnetic reconnection resulting from the Kelvin-Helmholtz instability in the shearing region (Sironi et al. 2020), and shock acceleration (Tavecchio 2021). In the latter case, these seed particles remain essentially uncooled for $\gtrsim 7.8 \times 10^{4} B_{-5}^{-2}(1+f)^{-1} \mathrm{yrs}$, and are responsible for the radio-tonear-infrared emissions from the jet. In this paper, we do not consider a specific acceleration mechanism for the seed particles, which might be one or more of the above mechanisms, as we are only interested in particles with higher energies accelerated through shear acceleration. Instead, we assume that seed particles are injected below a certain energy $\left(\gamma_{\mathrm{cr}}\right)$, so that we can take $Q(\gamma, t)=0$ for $\gamma>\gamma_{\mathrm{cr}}$ for the shear acceleration analysis in the following. Thus our solution only applies for particles with $\gamma>\gamma_{\mathrm{cr}}$, and is entirely independent of the particle distribution below $\gamma_{\mathrm{cr}}$.

As the dynamical timescale is larger than the acceleration timescale for large-scale jets and even for individual knots (see Eqs. (14-16) in Section 3.1), it is appropriate to take a quasi-steady-state approximation, i.e. $\partial n(\gamma, t) / \partial t=0$. In this case, we find that Eq. (8) has a general solution. The solution for $q=1$ is given in Eq. (A6). For $1<q \leq 2$, which is of more common astrophysical interest, the solution is (see Appendix A for a derivation),

$n(\gamma)=C_{+} \gamma^{s_{+}} F_{+}(\gamma, q)+C_{-} \gamma^{s_{-}} F_{-}(\gamma, q)$,

where the power-law spectral indices are

$s_{ \pm}=\frac{q-1}{2} \pm \sqrt{\frac{(5-q)^{2}}{4}+w}$,

where

$w=\frac{(6-q) \tau_{\mathrm{sh}}}{\tau_{\mathrm{esc}}}=\frac{4 c^{2}}{3 D_{\mathrm{sh}} \Delta r_{\mathrm{j}}^{2}}$

is a dimensionless measure of the shear viscosity. This indicates that the particle spectral hardness is mainly determined by the turbulence spectrum and the balance of acceleration and escape. For $1<q<2$ the indices satisfy $s_{+}>0$ and $s_{-}<0$.

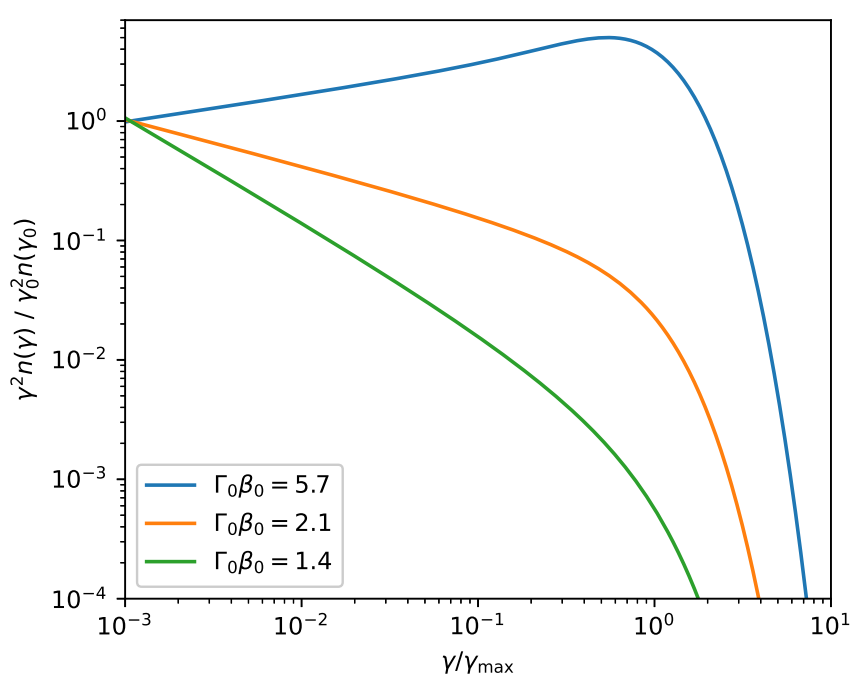

Figure 1. Three examples of particle energy distributions are calculated from Eqs. (9-13) with different velocities, i.e. $\Gamma_{0} \beta_{0}=1.4,2.1,5.7$, where $q=5 / 3$ is adopted. The spectra are normalized relative to their value at $\gamma_{0}=10^{-3} \gamma_{\max }$.

The $F_{ \pm}$are defined as

$F_{ \pm}(\gamma, q)={ }_{1} F_{1}\left[\frac{2+s_{ \pm}}{q-1}, \frac{2 s_{ \pm}}{q-1} ;-\frac{6-q}{q-1}\left(\frac{\gamma}{\gamma_{\max }}\right)^{q-1}\right]$,

where ${ }_{1} F_{1}$ is Kummer's confluent hypergeometric function (e.g., Abramowitz \& Stegun 1972). For negligible radiative losses, i.e. for $\gamma \ll \gamma_{\max }, F_{ \pm} \approx 1$, such that the solution resembles a power-law. The power-law index is consistent with previous results (Kimura et al. 2018; Rieger \& Duffy 2019).

The spectrum is obtained from the combination of two power-law components with the integration constants $\left(C_{ \pm}\right)$being determined by two requirements, i.e., the condition of $n \rightarrow 0$ for $\gamma \rightarrow \infty$ (see Appendix A) and the normalization of the spectrum. We find that the spectrum exhibits an exponential-like-cutoff power-law shape, where the power-law index is dominated by the $s_{-}$component.

The particle spectral index can be specifically determined for a given shearing profile. Here we consider a simple shearing profile with a jet speed linearly decreasing from $\beta_{\mathrm{j}}=\beta_{0}$ at $r_{\mathrm{j}}-\Delta r_{\mathrm{j}}$ to $\beta_{\mathrm{j}}=0$ at $r_{\mathrm{j}}$, where $r_{\mathrm{j}} \geq \Delta r_{\mathrm{j}}$ is the jet width. Performing a simple r-averaging, one obtains (see Eq. (9) in Rieger \& Duffy 2019)

$w=40 \ln ^{-2} \frac{\left(1+\beta_{0}\right)}{\left(1-\beta_{0}\right)}$.

For this profile and assuming $q=5 / 3$, we show three examples of the resulting particle distributions in Figure 1. As one can see, the spectral shape is highly dependent on the jet speed $\beta_{0}$. In the highly relativistic limit, we have $w \rightarrow 0$, and the spectrum with index $s_{-}=q-3$ is approached (e.g., Webb et al. 2018, 2019; Rieger \& Duffy 2019), in which particle escape will be insignificant, i.e. $\tau_{\mathrm{esc}} \gg \tau_{\mathrm{sh}}$. While for non-relativistic flow speeds, steep spectra are obtained. In general, the spectral index is sensitive to the employed velocity profile, with somewhat steeper shapes towards lower speeds being possible (Webb et al. 2019), while harder spectra may become possible if particles could re-enter the jet again (Webb et al. 2020). 


\section{RADIATION FROM ACCELERATED ELECTRONS IN LARGE-SCALE SHEARING JETS}

\subsection{General radiation features}

In the following we examine the capability of particle acceleration via the shear mechanism, and study its associated radiation features (cf. also Liu et al. 2017; Rieger \& Duffy 2019). As shown above, in steady-state the accelerated particles produce power-law spectra with an exponential-like cut-off. Such a steady state can only be achieved when the dynamical time $\left(\tau_{\mathrm{dyn}}\right)$ greatly exceeds the acceleration time. For trans-relativistic jets, the dynamical time can be expressed as

$\tau_{\text {dyn }}=L_{z} / \beta_{0} c=3.26 \times 10^{2} \chi r_{\mathrm{j}, 2} \beta_{0}^{-1} \mathrm{yrs}$,

where the length of the acceleration region is taken to be $L_{z}=\chi r_{\mathrm{j}}$, with $r_{\mathrm{j}}=10^{2} r_{\mathrm{j}, 2}$ pc. We set $\Lambda_{\max }=\xi_{2} \Delta r_{\mathrm{j}}$ such that the outer scale of the turbulence is less than the characteristic shearing scale-width. We define $\eta=\xi_{2}^{q-1} \xi_{1}^{-1}$, and from this point forward restrict our focus to Kolmogorov turbulence $q=5 / 3$, other cases being a trivial extension. The acceleration time is given numerically as

$\tau_{\mathrm{sh}}=1.38 \times 10^{3} w B_{-5}^{1 / 3} \Delta r_{\mathrm{j}, 2}^{4 / 3} \gamma_{9}^{-1 / 3} \eta^{-1} \mathrm{yrs}$,

where $\gamma=10^{9} \gamma_{9}$. We find that the condition for the application of the steady-state approximation is met when the ratio of jet radius to acceleration length satisfies

$\chi \gtrsim 4.2 w \beta_{0} B_{-5}^{1 / 3} \Delta r_{\mathrm{j}, 2}^{4 / 3} r_{\mathrm{j}, 2}^{-1} \gamma_{9}^{-1 / 3} \eta^{-1}$.

For large-scale AGN jets, this condition is likely to be satisfied. Even in jets where the X-ray emission is dominated by knotted structures, this criterion might be met on the scale of individual knots, at least marginally. Observations suggest that for typical knots $\chi>2 / \sin \theta$, where $\theta$ is the viewing angle to the jet axis, which can be small. For example, in the case of 3 C $273, \theta \lesssim 0.13$ has been reported (e.g. Meyer et al. 2016), which would lead to $\chi>16$.

The scattering timescale may also be expressed numerically as

$\tau_{\mathrm{sc}}=26.8 \Delta r_{\mathrm{j}, 2}^{2 / 3} \gamma_{9}^{1 / 3} \eta B_{-5}^{-1 / 3} \mathrm{yrs}$.

Here, to ensure particles are in fact accelerated, we require that at least one scattering time can occur, i.e. $\tau_{\text {sc }} \lesssim \tau_{\text {sh }}$. This leads to an additional constraint

$\eta \lesssim 7.17 w^{1 / 2} B_{-5}^{1 / 3} \Delta r_{\mathrm{j}, 2}^{1 / 3} \gamma_{9}^{-1 / 3}$.

The accelerated electrons suffer from radiation losses on a characteristic cooling time

$\tau_{\mathrm{c}}=2.45 \times 10^{2} B_{-5}^{-2}(1+f)^{-1} \gamma_{9}^{-1} \mathrm{yrs}$.

Acceleration proceeds provided $\tau_{\mathrm{sh}} \lesssim \tau_{\mathrm{c}}$, which translates to another condition

$\eta \gtrsim 5.61 w B_{-5}^{7 / 3}(1+f) \Delta r_{\mathrm{j}, 2}^{4 / 3} \gamma_{9}^{2 / 3}$.

The inequalities (18) and (20) further require $\gamma_{9} \lesssim 1.28 B_{-5}^{-2}(1+$ $f)^{-1} \Delta r_{\mathrm{j}, 2}^{-1} w^{-1 / 2}$, while the Larmor radius is $r_{\mathrm{L}, e}=0.05 \gamma_{9} B_{-5}^{-1} \mathrm{pc}$. Thus the Hillas criterion can be satisfied, and the corresponding maximum energy for electrons is

$E_{e, \max }=0.7 B_{-5}^{-2} \Delta r_{\mathrm{j}, 2}^{-1} w^{-1 / 2}(1+f)^{-1} \mathrm{PeV}$,

The resultant maximum synchrotron photon energy that the electrons may therefore radiate is

$E_{\gamma, \max }=82.3 B_{-5}^{-3} \Delta r_{\mathrm{j}, 2}^{-2} w^{-1}(1+f)^{-2} \mathrm{keV}$.
This demonstrates that electrons, energized via shear acceleration can produce X-rays. In large-scale jets, such X-rays are naturally expected to be emitted quasi-continuously along the jet. We now apply this model to selected observations of X-ray jets.

\subsection{Application to large-scale $\mathrm{X}$-ray jets}

Observations show that large-scale jets can be bright in X-rays even on scales of one-hundred-kpc (e.g. Harris \& Krawczynski 2006). Although the mechanism producing this X-ray radiation is still under debate, a synchrotron origin is strongly supported by current evidence e.g. the extended $\mathrm{TeV}$ radiation from Centaurus $\mathrm{A}$ (H. E. S. S. Collaboration et al. 2020), and the optical polarimetry and gamma-ray observations of 3C 273 (Meyer \& Georganopoulos 2014; Perlman et al. 2020). In the following, we take these two jets as examples. We show that their X-ray signatures can be explained in the framework of shear acceleration with typical jet parameters. Note the fitting parameters are exemplary, not unique. The SED modelling is performed using the NAIMA package (Zabalza 2015). This package includes tools to calculate the non-thermal radiation from relativistic particle populations and to perform Markov-Chain-Monte-Carlo fitting of the observed spectra. Here, we employ NAIMA to calculate the (one-zone) synchrotron and IC emission, and to model the lowenergy electron distribution, which is treated as independent input and assumed to be a (cut-off) power-law distribution for FR I (II) sources; the high-energy electron distribution is modelled with Eqs. $(9-13,21)$.

\subsubsection{FR I jet: Centaurus A}

Centaurus A (NGC 5128) is an FR I radio galaxy located at a distance of $3.8 \mathrm{Mpc}$ (Harris et al. 2010). Radio observations of the jet's proper motion on the sub-kpc scale suggest that the jet is moving with a mildly relativistic speed of $\beta_{0} \sim 0.5$ (Hardcastle et al. 2003; Snios et al. 2019). Recently, the High Energy Stereoscopic System (H.E.S.S.) has detected extended veryhigh-energy (VHE) emission from the large-scale jet of Centaurus A (H. E. S. S. Collaboration et al. 2020).

In Figure 2, we show an exemplary multi-wavelength-SED fit of the large-scale jet emission of Centaurus A. The data are taken from Hardcastle et al. (2006) and H. E. S. S. Collaboration et al. (2020). The electrons are assumed to follow a broken power-law spectrum with an exponential cut-off. We find that the radio-to-optical SED can be explained by synchrotron radiation from electrons with $N_{1}(E)=K_{1} E^{-\alpha_{1}}$ for $E_{\min , 1} \leq E \leq E_{\mathrm{b}}$ and $K_{1}$ being the normalization constant. The X-ray and $\mathrm{TeV}$ data, on the other hand, is modelled as synchrotron and IC radiation from shear accelerated electrons $\left(N_{2}(E)\right)$, i.e. for $E \geq E_{\mathrm{b}}$, we adopt Eqs. $(9-13,21)$, and the normalization is set to keep $N_{1}\left(E_{\mathrm{b}}\right)=N_{2}\left(E_{\mathrm{b}}\right)$. The model has in total 7 free parameters: $K_{1}, E_{\min , 1}, E_{\mathrm{b}}, \alpha_{1}, w, B, \Delta r_{\mathrm{j}}$. For the IC spectrum, the seed photons come from the CMB, starlight (Abdo et al. 2010), and the radiation from dust (Weiß et al. 2008). The seed photon energy density is found to be much smaller than that of the magnetic field, i.e. $f \ll 1$.

The model parameters are shown in Table 1 . The radio-tooptical fit is consistent with previous findings (Hardcastle et al. 2006; H. E. S. S. Collaboration et al. 2020). The X-ray and TeV spectra can be reproduced with $w=15.0, B=17.1 \mu \mathrm{G}$, and $\Delta r_{\mathrm{j}, 2}=1$ for our shear-acceleration model. This implies a spectral index of $s_{-}=-3.9$, a jet velocity at $\beta_{0} \approx 0.67$, and an electron energy cut-off at $57 \mathrm{TeV}$. The (spine) velocity is slightly larger than that estimated based on 


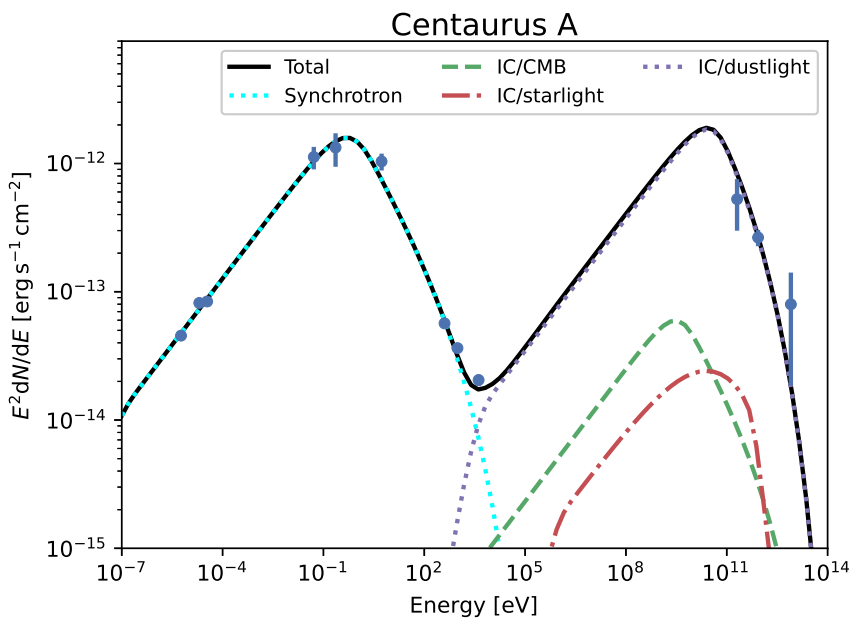

Figure 2. The multi-wavelength SED of Centaurus A reproduced by synchrotron and IC radiation of electrons with an exponential-cut-off broken power-law spectrum in the framework of shear acceleration. The model parameters are shown in Table 1.

radio observations. This difference is reasonable given the uncertainties in the shearing profile and the fact that the radio/optical emission may not fully trace the inner spine speed. From the morphology study in radio and X-ray band, the projected jet length is found to be a few $\mathrm{kpc}$, and the half jet width can be of order $\Delta r_{\mathrm{j}, 2} \sim 1$ (Hardcastle et al. 2003). Thus both the X-ray and TeV radiation can in theory be accounted for exclusively within a shear-acceleration model. The kinetic jet power can be estimated as $P_{\text {jet }}=\left(W_{e}+W_{p}+W_{B}\right) \beta c / L_{z}$ where $W_{e}, W_{p}, W_{B}$ is the total energies of relativistic electrons, cold protons, and magnetic field in the radiating region. Taking the jet length to be $L_{z} \sim 2 \mathrm{kpc}$, we obtain $P_{\text {jet }} \sim 3.7 \times 10^{42} \mathrm{erg} / \mathrm{s}$ for Cen A. This is much smaller than the Eddington luminosity of Cen A, which is $L_{\mathrm{Edd}} \sim 6.9 \times 10^{45} \mathrm{erg} / \mathrm{s}$ for a mass $5.5 \times 10^{7} M_{\odot}$ (Cappellari et al. 2009), and compatible with constraints on the average jet power inferred from modelling of the lobes in Cen A (Yang et al. 2012).

\subsubsection{FR II jet: $3 C 273$}

3C 273 is an FR II radio galaxy, located at a redshift of $z=0.158$. In general, FR II jets are dominated by bright knotted structures, which often exhibit very hard X-ray spectra with X-ray spectral indices comparable to their radio spectral indices. For 3C 273 only the first two knots have such hard X-ray spectra, while the X-ray spectra become softer for outer knots (Jester et al. 2006, see also our Figure 3 ). We here take the first two knots (A and B1) and an outer knot (C2) as examples. Below we show that the primary spectral features in the three knots can be naturally accounted for in the shear acceleration model. The radio, optical, and X-ray data are taken from Jester et al. (2007). The $\gamma$-ray upper limits are from Meyer \& Georganopoulos (2014).

In Figure 3, we show exemplary fits of knots A and B1, and $\mathrm{C} 2$, where the multi-wavelength SEDs are well reproduced. The radio-to-optical SED is explained by synchrotron radiation from electrons with $N_{1}(E)=K_{1} E^{-\alpha_{1}} \exp \left[-\left(E / E_{\mathrm{b}}\right)^{2}\right]$ for $E>E_{\min , 1}$, where $K_{1}$ is the normalization constant, and the super exponential cut-off follows from cooling effects (Zirakashvili \& Aharonian 2007). The X-ray SED is explained by synchrotron emission from shear accelerated electrons $\left(N_{2}(E)\right)$, which follows Eqs. (9-13,
21) with a normalization constant $K_{2}\left(C_{+}, C_{-}\right)$for $E>E_{\min , 2}$. Thus, two additional free parameters are necessary, i.e. we have $K_{1}, E_{\min , 1}, E_{\mathrm{b}}, \alpha_{1}, K_{2}, E_{\min , 2}, w, B, \Delta r_{\mathrm{j}}$. The IC/CMB radiation for the two electron populations are calculated with the extragalactic background light (EBL) absorption taken into account following Domínguez et al. (2011).

The model parameters are shown in Table 1. The radio-to-optical SEDs are consistent with a synchrotron interpretation as suggested by previous analysis (Jester et al. 2007). The UV-to-X-ray SED can be matched using $\Delta r_{\mathrm{j}, 2}=10, w=4.7, B=2.8 \mu \mathrm{G}(w=6.8$, $B=2.2 \mu \mathrm{G})$ for knots $\mathrm{A}+\mathrm{B} 1(\mathrm{C} 2)$ within the shear acceleration framework. This implies a spectral index of $s_{-}=-2.4(-2.8)$, a jet velocity at $\beta_{0} \approx 0.90(0.84)$, and an electron-energy cut-off at 370 (490) $\mathrm{TeV}$ for knots $\mathrm{A}+\mathrm{B} 1$ (C2). The jet speed is consistent with the upper-limit from proper-motion studies, which suggest $\Gamma_{0}<2.9$ for the knot bulk Lorentz factor (Meyer et al. 2016). From the Xray morphology, the jet width is $r_{\mathrm{j}} \sim 1 \mathrm{kpc}$ (Jester et al. 2006), and the length is $\sim 4 / \sin \theta(3 / \sin \theta)$ for knots $A+B 1$ (C2), where $\theta \sim 0.13$ (e.g., Meyer et al. 2016). The corresponding jet power is $2.7 \times 10^{45}\left(1.3 \times 10^{46}\right) \mathrm{erg} / \mathrm{s}$ for knots $\mathrm{A}+\mathrm{B} 1(\mathrm{C} 2)$. This is much smaller than the Eddington luminosity of $3 \mathrm{C} 273, L_{\text {Edd }} \sim 8.2 \times 10^{47}$, adopting a black hole mass of $6.6 \times 10^{9} M_{\odot}$ (Paltani \& Türler 2005).

\section{IMPLICATIONS FOR UHECR ACCELERATION}

AGN jets are proposed as potential ultra-high-energy cosmic-ray (UHECR) accelerators based on the Hillas criterion (Hillas 1984; Aharonian et al. 2002), although the acceleration mechanism(s) that might achieve such energies is an open question. As shown above, large-scale jets can in principle accelerate electrons up to PeV energies. We now study its potential for CR acceleration. To ensure that particles can be accelerated in trans-relativistic jets, the condition $\tau_{\text {dyn }}>\tau_{\text {sh }}$ must be satisfied, which requires seed nuclei to have energies

$E_{\text {seed nuclei }}>38 Z B_{-5} \Delta r_{\mathrm{j}, 2}^{4} w^{3} \beta_{0}^{3} \chi_{1}^{-3} \eta^{-3} r_{\mathrm{j}, 2}^{-3} \mathrm{TeV}$,

where $\chi_{1}=\chi / 10, Z$ is the atomic number, and $\eta \sim 1$ encapsulates the turbulent magnetic field strength and scales defined previously.

The maximum attainable energy is limited by several requirements. For consistency, we require $\tau_{\text {sc }} \lesssim \tau_{\text {sh }}$, form which we obtain

$E_{\text {nuclei }} \lesssim 0.2 Z B_{-5} \Delta r_{\mathrm{j}, 2} w^{3 / 2} \eta^{-3} \mathrm{EeV}$.

Cooling via synchrotron radiation is irrelevant for the fields we consider. We further note that the Larmor radius of a nucleus is $r_{\mathrm{L}}=0.1 \gamma_{9} B_{-5}^{-1} Z^{-1} \mathrm{kpc}$, which can be comparable to the size of the shearing region. Based on the Hillas criterion, we restrict the Larmor radius to be inside this region, i.e.,

$E_{\text {Hillas }} \leq 0.9 Z \beta_{0} B_{-5} \Delta r_{\mathrm{j}, 2} \mathrm{EeV}$.

Thus the maximum energy may therefore be expressed as

$E_{\max }=\min \left[0.9,0.2 w^{3 / 2} \eta^{-3}\right] Z B_{-5} \Delta r_{\mathrm{j}, 2} \mathrm{EeV}$.

For large-scale AGN jets, EeV protons are in theory achievable, while heavier nuclei would extend to higher energies. Note that for $\mathrm{EeV}$ protons, the characteristic synchrotron photon energy is $E_{p \text {,syn }} \approx$ $30 \gamma_{9}^{2} B_{-5} \mathrm{eV}$ (Aharonian 2002). We conclude that shear acceleration is a promising mechanism for the acceleration of UHECRs in largescale AGN jets (e.g., Liu et al. 2017; Kimura et al. 2018; Webb et al. 2018; Rieger \& Duffy 2019). 
Table 1. The exemplary parameters for the fitted SEDs of large-scale jets, Centaurus A and 3C 273. The low-energy component are responsible for radio-to-optical SED, while the high-energy component are responsible for the X-ray SED. The jet speed is calculated from Eq. (13).

\begin{tabular}{ccccc}
\hline $\begin{array}{c}\text { Source } \\
\text { name }\end{array}$ & $\begin{array}{c}\text { Low-energy component } \\
\left(E_{\mathrm{min}, 1}, E_{\mathrm{b}}, \alpha_{1}\right)\end{array}$ & $\begin{array}{c}\text { High-energy component } \\
\left(E_{\mathrm{min}, 2}, w, B, \Delta r_{\mathrm{j}, 2}\right)\end{array}$ & $\begin{array}{c}\text { Jet power in units of } \\
\text { erg/s and } L_{\mathrm{Edd}}\end{array}$ & $\begin{array}{c}\text { Jet } \\
\text { speed }\end{array}$ \\
\hline Centaurus A & $0.2 \mathrm{GeV}, 0.75 \mathrm{TeV},-2.31$ & $-, 15.0,17.1 \mu \mathrm{G}, 1$ & $3.7 \times 10^{42} \mathrm{erg} / \mathrm{s}, 5.4 \times 10^{-4} L_{\mathrm{Edd}, \mathrm{CenA}}$ & $0.67 c$ \\
\hline 3C 273 - Knots A+B1 & $1.5 \mathrm{GeV}, 1.1 \mathrm{TeV},-2.28$ & $2.5 \mathrm{TeV}, 4.7,2.8 \mu \mathrm{G}, 10$ & $2.7 \times 10^{45} \mathrm{erg} / \mathrm{s}, 3.2 \times 10^{-3} L_{\mathrm{Edd}, 3 \mathrm{C} 273}$ & $0.90 c$ \\
\hline 3C 273 - Knot C2 & $1.5 \mathrm{GeV}, 1.6 \mathrm{TeV},-2.52$ & $1.9 \mathrm{TeV}, 6.8,2.2 \mu \mathrm{G}, 10$ & $1.3 \times 10^{46} \mathrm{erg} / \mathrm{s}, 1.5 \times 10^{-2} L_{\mathrm{Edd}, 3 \mathrm{C} 273}$ & $0.84 c$ \\
\hline
\end{tabular}
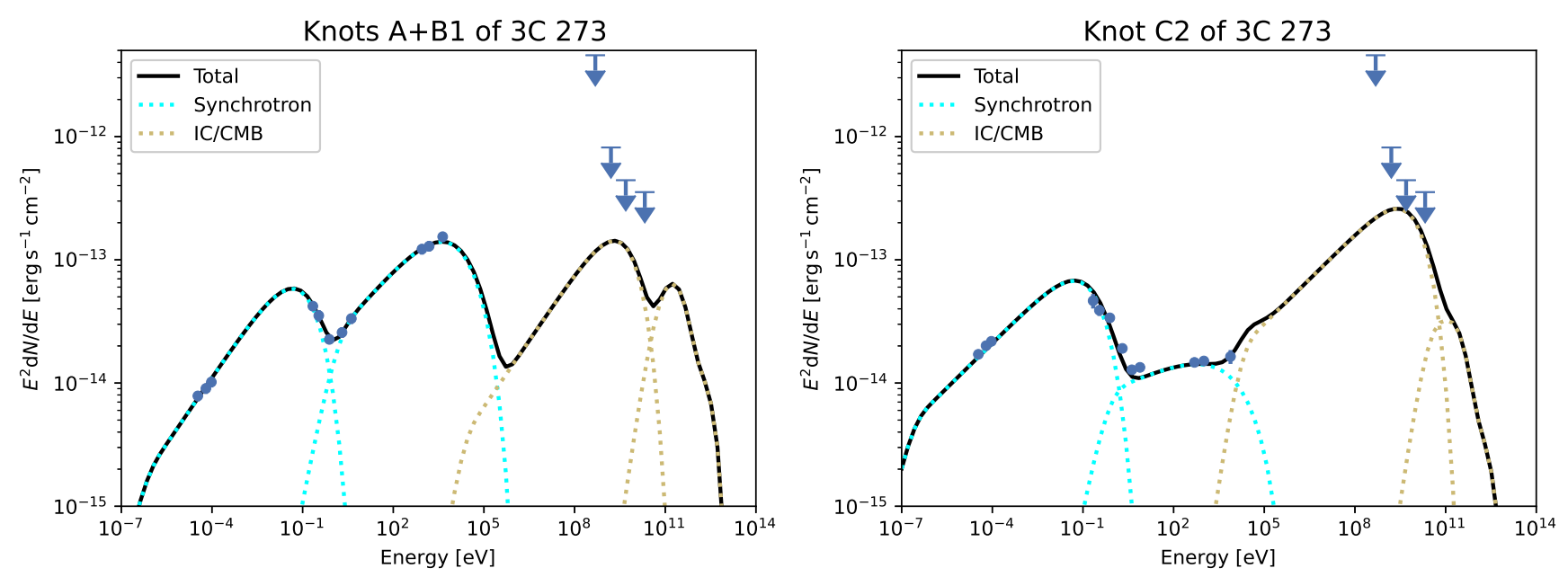

Figure 3. The multi-wavelength SED of the knots in 3C 273 (left panel for Knots A+B1 and right panel for knot C2) reproduced by synchrotron and IC radiation of two electron populations with exponential-cut-off power-law spectra in the framework of shear acceleration. The model parameters are shown in Table 1.

The accelerated proton spectrum within the jet should approximately follow Eq. (9). On the other hand, the spectrum of the escaping protons, which can contribute to the observed CRs, follows

$\dot{n}_{\mathrm{esc}} \propto \gamma^{s_{-}} / \tau_{\mathrm{esc}}=\gamma^{2-q+s_{-}}$.

This spectrum is harder than the confined particle spectrum. The Xray observations suggest spectral indices of $\left|s_{-}\right| \approx 3.6$ for Centaurus A and 2.4(2.8) for 3C 273, such that the implied index for escaping protons is 3.3 for Centaurus A and 2.1(2.5) for 3C 273.

\section{SUMMARY AND CONCLUSION}

In this paper, we have explored the capability of gradual shear flow particle acceleration in large-scale X-ray jets. Velocity-shear stratification is naturally expected in large-scale jets, and energetic seed particles can be accelerated by interacting with the magnetic field inhomogeneities frozen in stratified layers. The seed particles can be injected from shock acceleration, magnetic reconnection, and/or stochastic acceleration. We provide a general solution of the steady-state Fokker-Planck equation for shear acceleration in transrelativistic jets. In general, the accelerated particle spectrum resembles an exponential-like-cutoff-power-law spectrum. The power-law index is essentially determined by the turbulence spectrum, and the balance of escape and acceleration of particles (see Eq. 10). Assuming a simple linearly decreasing velocity profile in the boundary of large-scale jets, the particle spectrum can be determined (Eq. 13). The maximum energy of the electrons is typically found to be $\sim \mathrm{PeV}$, radiating $\sim \mathrm{keV} \mathrm{X}$-rays via synchrotron radiation. Such synchrotron origin of the X-ray emission in knots of powerful jets has also recently been investigated by Tavecchio (2021), where shear acceleration of a locally produced shock-accelerated particle population is explored numerically.

We have applied our model to observations of the X-ray jets in Centaurus A and 3C 273. In FR I jets, the radio-optical-X-ray spectrum usually conforms to synchrotron radiation from electrons with a broken-power-law spectrum. For Centaurus A, the recent TeV observations further support the synchrotron origin of X-rays. In FR II jets, the optical polarimetry and $\gamma$-ray observations disfavor the popular IC origin of X-rays, while electron or proton synchrotron remain viable. We note however that proton synchrotron in X-rays usually requires $\sim \mathrm{mG}$ magnetic fields in kpc-scale jets (Aharonian 2002; Wang et al. 2020). Modelling of the radiative features (e.g. Sikora et al. 2005; Potter 2017) as well as MHD simulations (e.g. Chatterjee et al. 2019) both suggest that the jet is kinetic energy dominated on such scales. Therefore, an electron synchrotron origin of the X-ray emission is favored for both FR I and II jets. To explain the extended X-ray emission at kpc-scale jets, an in-situ (re-)acceleration mechanism is required due to the significant synchrotron radiation cooling. In contrast to shock acceleration, which would result in the most energetic electrons being localised to the position of a shock, and magnetic reconnection or related processes, which are only effective in magnetically dominated jets at $\lesssim$ pc scale (Matthews et al. 2020), we find shear acceleration can naturally accelerate particles along the jet even at $>\mathrm{kpc}$ scales. In our shear-acceleration model, the multi-wavelength SED (Figures 2 and 3) can be satisfactorily reproduced by synchrotron and IC radiation with typical jet parameters (Table 1) for Centaurus A and 3C 273. The required jet power in our 
exemplary fittings is significantly below the respective Eddington Luminosity of each source, with $P_{\text {jet }} \sim 4 \times 10^{42} \mathrm{erg} / \mathrm{s}$ for Centaurus A and $P_{\text {jet }} \sim 10^{45}-10^{46} \mathrm{erg} / \mathrm{s}$ for $3 \mathrm{C} 273$. This, as well as the derived jet velocities at $\sim 0.67 c$ for Centaurus $\mathrm{A}$ and $\sim 0.9 c(0.84 c)$ for the knots $\mathrm{A}+\mathrm{B} 1(\mathrm{C} 2)$ of $3 \mathrm{C} 273$, is consistent with the general picture of FR II jets being more powerful than FR I jets. Further, the decelerating jet of 3C 273, which in our model should decrease from $\sim 0.9 c$ at knots $\mathrm{A}+\mathrm{B} 1$ to $\sim 0.84 c$ at knot $\mathrm{C} 2$, is also naturally expected as the jet propagates. We note that two populations of electrons are required in 3 C 273, which may hint that the radio-to-optical and X-ray SEDs are produced in different locations, for example in the spine and sheath picture (e.g., Jester et al. 2006). However, only one population of electrons is needed in Centaurus A. This difference may relate to jet dynamics, such as the properties of the shearing region, and should be followed up in future work.

For shear acceleration in large-scale jets, protons and other nuclei can also be accelerated with a spectrum similar to that of electrons, while the escaping particles could have an even harder spectrum. The maximum energy is found to be $E_{\max }=$ $\min \left[0.9,0.2 w^{3 / 2} \eta^{-3}\right] Z B_{-5} \Delta r_{\mathrm{j}, 2} \mathrm{EeV}$, consistent with previous findings (Liu et al. 2017; Rieger \& Duffy 2019). This work further supports the evidence that shear acceleration can provide a favourable mechanism for UHECR acceleration in large-scale jets.

\section{ACKNOWLEDGEMENTS}

We thank the referee for valuable comments, and X. N. Sun, Y. Mizuno and M. Tsirou for helpful discussions. J.S.W. was supported by China Postdoctoral Science Foundation. R.-Y. L. is supported by NSFC No. U2031105.

\section{DATA AVAILABILITY}

No new data were generated or analysed in support of this research.

\section{REFERENCES}

Abdo A. A., et al., 2010, Science, 328, 725

Abramowitz M., Stegun I. A., 1972, Handbook of Mathematical Functions Aharonian F. A., 2002, MNRAS, 332, 215

Aharonian F. A., Belyanin A. A., Derishev E. V., Kocharovsky V. V., Kocharovsky V. V., 2002, Phys. Rev. D, 66, 023005

Begelman M. C., 1998, ApJ, 493, 291

Bell A. R., 2013, Astroparticle Physics, 43, 56

Berezhko E. G., 1981, ZhETF Pisma Redaktsiiu, 33, 416

Berezhko E. G., Krymskii G. F., 1981, Soviet Astronomy Letters, 7, 352

Blandford R., Eichler D., 1987, Phys. Rep., 154, 1

Blandford R., Meier D., Readhead A., 2019, ARA\&A, 57, 467

Boccardi B., Krichbaum T. P., Bach U., Mertens F., Ros E., Alef W., Zensus J. A., 2016, A\&A, 585, A33

Breiding P., Meyer E. T., Georganopoulos M., Keenan M. E., DeNigris N. S., Hewitt J., 2017, ApJ, 849, 95

Cappellari M., Neumayer N., Reunanen J., van der Werf P. P., de Zeeuw P. T., Rix H. W., 2009, MNRAS, 394, 660

Cara M., et al., 2013, ApJ, 773, 186

Celotti A., Ghisellini G., Chiaberge M., 2001, MNRAS, 321, L1

Chatterjee K., Liska M., Tchekhovskoy A., Markoff S. B., 2019, MNRAS, 490, 2200

Domínguez A., et al., 2011, MNRAS, 410, 2556

Drury L. O., 1983, Reports on Progress in Physics, 46, 973

Earl J. A., Jokipii J. R., Morfill G., 1988, ApJ, 331, L91

Fanaroff B. L., Riley J. M., 1974, MNRAS, 167, 31P
Gabuzda D. C., Reichstein A. R., O’Neill E. L., 2014, MNRAS, 444, 172

Georganopoulos M., Meyer E., Perlman E., 2016, Galaxies, 4, 65

H. E. S. S. Collaboration et al., 2020, Nature, 582, 356

Hardcastle M. J., Birkinshaw M., Worrall D. M., 2001, MNRAS, 326, 1499

Hardcastle M. J., Worrall D. M., Kraft R. P., Forman W. R., Jones C., Murray S. S., 2003, ApJ, 593, 169

Hardcastle M. J., Kraft R. P., Worrall D. M., 2006, MNRAS, 368, L15

Harris D. E., Krawczynski H., 2006, ARA\&A, 44, 463

Harris G. L. H., Rejkuba M., Harris W. E., 2010, Publ. Astron. Soc. Australia, 27,457

Hillas A. M., 1984, ARA\&A, 22, 425

Jester S., Harris D. E., Marshall H. L., Meisenheimer K., 2006, ApJ, 648, 900

Jester S., Meisenheimer K., Martel A. R., Perlman E. S., Sparks W. B., 2007, MNRAS, 380, 828

Kataoka J., Stawarz Ł., Aharonian F., Takahara F., Ostrowski M., Edwards P. G., 2006, ApJ, 641, 158

Kim J., Balsara D. S., Lyutikov M., Komissarov S. S., 2018, MNRAS, 474, 3954

Kimura S. S., Murase K., Zhang B. T., 2018, Phys. Rev. D, 97, 023026

Kraft R. P., Forman W. R., Jones C., Murray S. S., Hardcastle M. J., Worrall D. M., 2002, ApJ, 569, 54

Laing R. A., Bridle A. H., 2014, MNRAS, 437, 3405

Liu R.-Y., Rieger F. M., Aharonian F. A., 2017, ApJ, 842, 39

Matthews J. H., Bell A. R., Blundell K. M., 2020, New Astron. Rev., 89,101543

Meyer E. T., Georganopoulos M., 2014, ApJ, 780, L27

Meyer E. T., Georganopoulos M., Sparks W. B., Godfrey L., Lovell J. E. J., Perlman E., 2015, ApJ, 805, 154

Meyer E. T., et al., 2016, ApJ, 818, 195

Mizuno Y., 2013, ApJS, 205, 7

Nagai H., et al., 2014, ApJ, 785, 53

O’Sullivan S., Reville B., Taylor A. M., 2009, MNRAS, 400, 248

Paltani S., Türler M., 2005, A\&A, 435, 811

Perlman E. S., Biretta J. A., Sparks W. B., Macchetto F. D., Leahy J. P., 2001, ApJ, 551, 206

Perlman E. S., Clautice D., Avachat S., Cara M., Sparks W. B., Georganopoulos M., Meyer E., 2020, Galaxies, 8, 71

Potter W. J., 2017, MNRAS, 465, 337

Rieger F. M., 2019, Galaxies, 7, 78

Rieger F. M., Duffy P., 2004, ApJ, 617, 155

Rieger F. M., Duffy P., 2006, ApJ, 652, 1044

Rieger F. M., Duffy P., 2016, ApJ, 833, 34

Rieger F. M., Duffy P., 2019, ApJ, 886, L26

Rieger F. M., Duffy P., 2021, ApJ, 907, L2

Rieger F. M., Bosch-Ramon V., Duffy P., 2007, Ap\&SS, 309, 119

Schlickeiser R., 2002, Cosmic Ray Astrophysics

Schwartz D. A., et al., 2014, Proceedings of the International Astronomical Union, 10, 219-224

Sikora M., Begelman M. C., Madejski G. M., Lasota J.-P., 2005, ApJ, 625, 72

Sironi L., Spitkovsky A., 2014, ApJ, 783, L21

Sironi L., Rowan M. E., Narayan R., 2020, arXiv e-prints, p. arXiv:2009.11877

Snios B., et al., 2019, ApJ, 871, 248

Stawarz Ł., Ostrowski M., 2002, ApJ, 578, 763

Sun X.-N., Yang R.-Z., Rieger F. M., Liu R.-Y., Aharonian F., 2018, A\&A, 612, A106

Tavecchio F., 2021, MNRAS, 501, 6199

Tavecchio F., Maraschi L., Sambruna R. M., Urry C. M., 2000, ApJ, 544, L23

Uchiyama Y., 2008, International Journal of Modern Physics D, 17, 1475

Wang Z.-J., Zhang J., Sun X.-N., Liang E.-W., 2020, ApJ, 893, 41

Webb G. M., 1989, ApJ, 340, 1112

Webb G. M., Barghouty A. F., Hu Q., le Roux J. A., 2018, ApJ, 855, 31

Webb G. M., Al-Nussirat S., Mostafavi P., Barghouty A. F., Li G., le Roux J. A., Zank G. P., 2019, ApJ, 881, 123

Webb G. M., Mostafavi P., Al-Nussirat S., Barghouty A. F., Li G., le Roux J. A., Zank G. P., 2020, ApJ, 894, 95

Weiß A., Kovács A., Güsten R., Menten K. M., Schuller F., Siringo G., Kreysa E., 2008, A\&A, 490, 77 


\section{$8 \quad$ Wang et al.}

Werner G. R., Uzdensky D. A., Cerutti B., Nalewajko K., Begelman M. C., 2016, ApJ, 816, L8

Yang R. Z., Sahakyan N., de Ona Wilhelmi E., Aharonian F., Rieger F., 2012, A\&A, 542, A19

Zabalza V., 2015, Proc. of International Cosmic Ray Conference 2015, p. 922

Zirakashvili V. N., Aharonian F., 2007, A\&A, 465, 695 


\section{APPENDIX A: DERIVATION OF STEADY-STATE SOLUTION FOR EQUATION (8)}

We here present a brief derivation of Eq. (9). Starting from Eq. (8) we seek a steady-state $(\partial n(\gamma, t) / \partial t=0)$ solution above some minimum injection energy, i.e. $Q\left(\gamma>\gamma_{\mathrm{cr}}, t\right)=0$ where $\gamma_{\mathrm{cr}}$ is the maximum energy particles injected into the jet. We seek solutions of the form $n(\gamma) \propto \gamma^{s} j(\gamma)$. Using $w=-6+2 q+\left(1-q+s_{ \pm}\right) s_{ \pm}$and setting $n(\gamma) \propto \gamma^{s_{ \pm}} j_{ \pm}(\gamma)$, Eq. (8) can be reduced to

$2\left(A_{2} / A_{1}\right)\left(s_{ \pm}+2\right) \gamma^{q-3} j_{ \pm}(\gamma)+\left[\gamma^{-1}\left(2-q+2 s_{ \pm}\right)+2\left(A_{2} / A_{1}\right) \gamma^{q-2}\right] j_{ \pm}^{\prime}(\gamma)+j_{ \pm}^{\prime \prime}(\gamma)=0$.

For $1<q \leq 2$, we can introduce a new variable $z=-\frac{6-q}{q-1}\left(\gamma / \gamma_{\max }\right)^{q-1}$ and its inverse $\gamma(z)=\left[(1-q) A_{1} z /\left(2 A_{2}\right)\right]^{1 /(q-1)}$. Changing to $z$ as the dependent variable, the above equation can be simplified to

$z \frac{d^{2} j_{ \pm}}{d z^{2}}+\left(b_{ \pm}-z\right) \frac{d j_{ \pm}}{d z}-a_{ \pm} j_{ \pm}=0$

where $b_{ \pm}=2 s_{ \pm} /(q-1)$ and $a_{ \pm}=\left(2+s_{ \pm}\right) /(q-1)$. This is Kummer's differential equation (e.g., Abramowitz \& Stegun 1972) which, provided $b$ is not a non-positive integer, has two independent solutions, which can be expressed in terms of the confluent hyper-geometric function as ${ }_{1} F_{1}\left(a_{ \pm}, b_{ \pm} ; z\right)$ and $z^{1-b_{ \pm}} F_{1}\left(1+a_{ \pm}-b_{ \pm}, 2-b_{ \pm} ; z\right)$.

It is readily shown that the four solutions are not linearly independent since (for fixed $w, q$ )

$\gamma^{S_{\mp}}{ }_{1} F_{1}\left(a_{\mp}, b_{\mp} ; z\right) \propto \gamma^{S_{ \pm}} z^{1-b_{ \pm}}{ }_{1} F_{1}\left(1+a_{ \pm}-b_{ \pm}, 2-b_{ \pm} ; z\right)$,

and thus only 2 solutions need be retained, as is expected.

The general solution may be expressed simply as

$n(\gamma)=C_{+} \gamma^{s_{+}}{ }_{1} F_{1}\left(a_{+}, b_{+} ; z\right)+C_{-} \gamma^{s_{-}}{ }_{1} F_{1}\left(a_{-}, b_{-} ; z\right)$.

For physically meaningful solutions, we require $n\left(\gamma \gg \gamma_{\max }\right) \rightarrow 0$. Using the asymptotic expression for large $z$ of the confluent hypergeometric function it follows that the solution decays as a power law $\propto \gamma^{-2}$ unless

$\frac{C_{+} \Gamma\left(b_{+}\right)}{\Gamma\left(b_{+}-a_{+}\right)} \gamma^{s_{+}}|z|^{a_{+}}+\frac{C_{-} \Gamma\left(b_{-}\right)}{\Gamma\left(b_{-}-a_{-}\right)} \gamma^{s_{-}}|z|^{a_{-}}=0$

where $\Gamma(x)$ is the Gamma function. With this condition, the solution cuts off exponentially above $\gamma_{\max }$. This completes the derivation.

Finally, we consider the singular case of $q=1$, for which the solution is easily shown to be

$n(\gamma)=C_{1} \gamma^{\sqrt{w+\left(2-A_{2} / A_{1}\right)^{2}}-A_{2} / A_{1}}+C_{2} \gamma^{-\sqrt{w+\left(2-A_{2} / A_{1}\right)^{2}}-A_{2} / A_{1}}$.

Note that in this case the radiation-limited maximum Lorentz factor cannot be defined, e.g. see Eq. (6). Although as discussed in the text, the solution is not meaningful when $r_{\mathrm{L}}>\Lambda_{\max }$.

This paper has been typeset from a $\mathrm{T}_{\mathrm{E}} \mathrm{X} / \mathrm{L} \mathrm{T} \mathrm{E} \mathrm{X}$ file prepared by the author. 